\title{
Políticas de avaliação do ensino naval
}

\author{
Natália Morais Corrêa Borges de Aguiar* \\ Maria Judith Sucupira da Costa Lins**
}

\section{Resumo}

0 presente artigo trata de um estudo de caso referente à avaliação do ensino naval e analisa contribuições de uma experiência de avaliação institucional na Marinha do Brasil. São analisadas as contribuições da Metodologia de Avaliação do Sistema de Ensino Naval, numa perspectiva comparativa dos resultados alcançados em três anos de sua implementação no Centro de Instituição Almirante Sylvio de Camargo (CIASC). A pesquisa analisa a avaliação institucional e os conceitos de meta-avaliação e de "cultura de avaliação" e sua fundamentação teórica são estudos de Penna Firme e Letichevsky (2002), Dias Sobrinho (2000) e Ristoff $(1996,2000)$. A partir das análises feitas, concluímos que a qualidade do ensino naval tem melhorado com o alcance progressivo de pontuações mais elevadas e verificamos que reuniões para discussão dos assuntos referentes à avaliação tornaram-se mais frequentes na organização de ensino, nas quais foram estabelecidas metas para a melhoria contínua em busca da excelência. Esta pesquisa abre caminhos para a construção de novos modelos de avaliação institucional, adaptados às distintas realidades acadêmicas.

Palavras-chave: Meta-avaliação. Ensino naval. Ensino.

\section{Introdução}

0 ensino na Marinha do Brasil (MB) é regido por lei particular e possui características próprias. Este ensino caracteriza-se como um processo contínuo e progressivo de educação, atualizado e aprimorado constantemente e tem como objetivo "prover ao pessoal da Marinha o conhecimento básico, profissional e militarnaval necessário ao cumprimento de sua missão constitucional" (BRASIL, 2006).

Entendemos que o aperfeiçoamento das práticas avaliativas adotadas na MB é de extrema importância para o cumprimento da sua missão e para a garantia da contínua melhoria dos estabelecimentos militares de ensino. Verificamos que a garantia do padrão de qualidade, a avaliação integral e contínua, e o efetivo aproveitamento da qualificação adquirida, em prol da Instituição, são principios basilares estabelecidos em sua lei de ensino. Portanto, argumentamos que é preciso avaliar a praticada avaliação do ensino feita na $M B$, ou seja, fazer uso da meta-

* Doutora em Educação (UFRJ), Oficial Pedagoga da Marinha do Brasil. E-mail: nmcba@predialnet.com.br.

** Doutora em Educação (UFRJ), Professora Associada da Faculdade de Educação da UFRJ.

E-mail: mariasucupiralins@terra.com.br. 
avaliação para melhoria das práticas, processos, métodos e instrumentos avaliativos vigentes na instituição militar.

A trajetória da avaliação educacional na MB apresenta três momentos distintos. Em um primeiro momento, as Organizações Militares (OM) de ensino utilizavam modelos próprios para avaliar seus cursos, sem uma visão sistêmica. 0 segundo momento foi marcado pela construção da primeira Sistemática de Avaliação do Sistema de Ensino Naval (SEN), mais conhecida como SAVSEN, que foi adotada pela maioria das OM de ensino do SEN.

Constatada a necessidade de alterações na primeira sistemática de avaliação, processo natural após dez anos de sua criação, a Diretoria de Ensino da Marinha (DEnsM), órgão central do SEN, a quem compete exercer a orientação normativa, a supervisão funcional e a fiscalização específica das organizações de execução do ensino, elaborou um novo método de avaliação que veio a substituir a sistemática SAVSEN, marcando assim, o terceiro momento da avaliação na MB.

Cabe à DEnsM a responsabilidade de supervisionar o cumprimento das políticas de avaliação e educacionais vigentes na MB. Em 2005, foi criado um Manual de Avaliação do Sistema de Ensino Naval - DEnsM-2001, que propõe uma metodologia de avaliação mais ampla e abrangente. Esta nova metodologia passou a ser utilizada em 2006 para avaliar a qualidade do ensino ministrado nas distintas OM de ensino.

A relevância deste estudo está na possibilidade de a MB, realizar a meta-avaliação, defendida por Penna Firme e Letichevsky (2002), ao avaliarmos a contribuição da avaliação normatizada pela DEnsM, verificando sua utilidade para a melhoria do ensino, ao analisarmos a evolução dos resultados alcançados historicamente pelo Centro de Instrução Almirante Sylvio de Camargo (CIASC), avançando na questão da avaliação contínua e contribuindo para o aperfeiçoamento e a melhoria da formação de seu pessoal. Além disso, o estudo irá contribuir para o amadurecimento de discussões sobre políticas de avaliação do ensino e sobre experiências e práticas avaliativas.

Verificamos que o Manual DEnsM-2001 detalha os procedimentos a serem adotados no âmbito do SEN e reforça a necessidade da autoavaliação dos estabelecimentos de ensino, conforme abaixo.

A realização da autoavaliação da gestão do ensino em cada organização torna possível o autoconhecimento por parte do estabelecimento de ensino, permitindo a identificação de seus fatores de força e fraqueza, representando, no caso do SEN, o primeiro passo para que sejam formuladas propostas e realizadas ações no sentido do aprimoramento do ensino na MB e da capacitação oferecida (BRASIL, 2011, p.1-1). 
Nesse sentido, o problema pesquisado consistiu em verificar se a metodologia de avaliação utilizada no CIASC, estabelecimento de ensino situado no Rio de Janeiro, subordinado ao Comando do Pessoal de Fuzileiros Navais (CPesFN) e escolhido para a realização da pesquisa, tem contribuído para o contínuo aperfeiçoamento do desempenho acadêmico e do planejamento da gestão da $0 \mathrm{M}$.

O CIASC, responsável pelos cursos de carreira de todos os Oficiais e Praças Fuzileiros Navais, vem aplicando a metodologia de avaliação do SEN desde 2006, mas é preciso verificar se o estabelecimento de ensino tem conseguido progressos e definido novos rumos a serem percorridos, numa perspectiva de avaliação contínua, conforme preceitua 0 Manual de Avaliação do SEN e autores como, Dias Sobrinho (2000) e Ristoff (1996, 2000).

\section{Objetivos e questões do estudo}

Os objetivos desta pesquisa foram analisar as contribuições da nova metodologia de avaliação para o SEN; comparar os resultados da avaliação do ensino, de 2007 a 2009, alcançados pelo CIASC; e relacionar as ações decorrentes e metas estabelecidas pelo Centro de Instrução em busca da excelência do ensino. As questões que nortearam o estudo foram:

a) comparando os resultados da avaliação global do CIASC, podemos diagnosticar avanços e melhorias na qualidade do ensino e da gestão da instituição; e

b) a partir dos resultados da avaliação, quais foram as principais ações decorrentes da OM de ensino avaliada em busca da excelência do ensino?

\section{Metodologia}

0 trabalho trata de um estudo de caso referente à avaliação institucional no CIASC, referendado por pesquisa bibliográfica e pesquisa documental sobre 0 assunto em tela. Além da revisão da literatura, na qual se discutiu os conceitos de avaliação, explícitos nas publicações que regulam o assunto na MB e nos trabalhos publicados por autores contemporâneos da educação, foram, também, analisados os resultados e as ações decorrentes da avaliação, apresentados nos Relatórios de Avaliação e demais documentos oficiais do Centro de Instrução.

\section{Revisão da literatura}

A avaliação é um ato cotidiano, intencional, e que se aplica a qualquer prática. 0 ato de avaliar implica em fazer balanços dos acertos e erros, dos avanços e limitações, daquilo que precisamos manter como está e daquilo que precisamos mudar para evoluir. Portanto, é preciso ter clareza do que se tem e do que se quer. A avaliação requer juizos de valor como declara Luckesi (1995). 0 autor entende avaliação como "um juizo de qualidade sobre dados relevantes, tendo em vista uma tomada de decisão" (LUCKESI, 1995, p. 69). 
Evidenciamos no Manual de Avaliação da MB (DEnsM-2001) um conceito similar em relação ao julgamento de valor, quando estabelece que o processo de avaliação caracteriza-se como um "juizo de valor, a partir de padrões, especificações ou requisitos previamente definidos, a fim de que se possa determinar o grau em que a qualidade do produto satisfaz a necessidade do usuário" (BRASIL, 2011, p.1-1).

A clareza e a transparência dos critérios avaliativos são de grande importância para qualquer política de avaliação institucional porque se define o que se entende por qualidade. Isso está previsto na publicação DEnsM-2001 e cabe aos estabelecimentos de ensino, conhecer, estudar e perseguir o alcance dos padrões de excelência estabelecidos pela MB. As definições dos critérios avaliativos estão expressos nos Roteiros de Avaliação (RotA) de cada OM de ensino do SEN.

A DEnsM estabeleceu categorias, indicadores e padrões para as cinco dimensões da avaliação previstas no Manual DEnsM-2001 - Corpo Docente, Corpo Discente, Organização Didático-Pedagógica, Instalações e Pós-Escolar. Identificamos que há uma clareza do que se quer, pois os parâmetros são divulgados e definidos a priori. Segundo Canen,

os critérios para o julgamento de valor partem de uma filosofia, de uma ideologia, de uma visão de mundo quanto ao que se espera da educação, que perfil de alunos e futuros profissionais desejamos formar e como julgar as ações dos atores e instituições educacionais para concretizar tais objetivos (CANEN, 2005, p. 97).

Vários estudiosos da educação ressaltam a importância de se investigar 0 processo avaliativo. Discussões acerca das concepções de avaliação e experiências institucionais estão presentes nos espaços acadêmicos e sociais, onde há uma tensão entre o que se tem e o que se espera ter, entre o real e o ideal.

A importância da continuidade do processo avaliativo tem sido amplamente defendida por estudiosos do tema avaliação. Dias Sobrinho (2000, p. 65) argumenta que a avaliação institucional não deve "se extinguir com a elaboração de relatórios e de diagnósticos, com o julgamento de resultados e ações já cumpridas", porque assim se transformará numa avaliação contemplativa, sem provocar mudanças da situação pesquisada e/ou avaliada.

De acordo com Ristoff $(1996,2000)$, um programa de avaliação deve ser contínuo. 0 princípio da continuidade, recomendado por ele, irá permitir a comparabilidade dos dados de um determinado momento ao outro, revelando o grau de eficácia das medidas adotadas a partir dos resultados obtidos. Para o autor, só a continuidade irá garantir a construção da "cultura de avaliação". Essa continuidade do processo avaliativo e da busca de melhores resultados deve ser incentivada e perseguida 
por todos os elementos participantes do processo avaliativo. Cultura de avaliação pode ser descrita como,

um conjunto de valores acadêmicos, atitudes e formas coletivas de conduta que tornem o ato avaliativo parte integrante do exercício diuturno de nossas funções. E este processo só logrará êxito se for coletivamente construido e se puder contar com intensa participação de seus membros, tanto nos procedimentos e implementação, como na utilização dos resultados. (RISTOFF, 2000).

Para que a avaliação seja útil e provoque mudanças, é preciso que os resultados da avaliação sejam discutidos, comparados periodicamente, para o constante estabelecimento de metas e estratégias de melhoria, numa busca incessante pelos padrões de excelência. Patton (1986, apud PENNA FIRME e LETICHEVSKY, 2002, p. 290) destaca que "o valor de uma avaliação é refletido na utilização de seus resultados". Essa cultura da avaliação está sendo disseminada no Manual DEnsM-2001, quando se estabelece que "é necessário criar, em todos os níveis, a consciência de que a avaliação é uma necessidade para qualquer esforço de melhoria" (BRASIL, 2011, p.1-1).

No Manual DEnsM-2001 ressalta-se que a avaliação institucional busca a superação das dificuldades com base na implementação de mudanças que "conduzam a um processo de permanente aprimoramento, a partir da compreensão de que ensinar, aprender e avaliar devem constituir um processo interativo contínuo" (BRASIL, 2011, p.1-1). A avaliação, portanto, nunca se esgota.

0 momento final do processo de avaliação institucional pode ser chamado de reavaliação. É um momento de sintese, que retoma criticamente os principais aspectos do processo vivido, que identifica as divergências e convergências relativas à auto-avaliação e à avaliação externa e consolida as principais metas a serem cumpridas [...] (DIAS SOBRINHO, 2000, p. 83).

A própria avaliação pode ser reavaliada. Essa prática é chamada de meta-avaliação, definida por Penna Firme e Letichevsky (2002, p. 289) como a "avaliação da avaliação". É considerada como uma atividade crucial e tem como propósito assegurar

a qualidade da avaliação que, por sua vez, tem na sua essência julgar o mérito e a relevância de seu foco de atenção, através de um processo de indagação sistemática - o mérito no que se refere às qualidades intrínsecas do objeto e a relevância no que se refere ao impacto e à repercussão do objeto-foco em questão (PENNA FIRME; LETICHEVSKY, 2002, p. 291). 
De acordo com Yarbrough et al. (2010), os estudiosos do tema metaavaliação, participantes do Joint Committee on Standards for Educacional Evaluation (1994), estabeleceram quatro padrões para conceituar o que é uma avaliação de qualidade: utilidade, viabilidade, ética e precisão. Esses padrões, até o momento, representam a diretriz mais utilizada para julgar a qualidade de uma avaliação conduzida em uma instituição.

Neste estudo, ao fazer uso da meta-avaliação, optamos pela categoria utilidade para verificar se a aplicação do Manual DEnsM-2001 tem contribuído para o alcance de melhorias e a busca pela excelência do ensino no Centro de Instrução Almirante Sylvio de Camargo (CIASC). De acordo com Worthen et al. (2004), a utilidade é apresentada em primeiro lugar na avaliação de programas, pois "o Joint Committee reconheceu que sem utilidade, uma avaliação de programa não presta, por melhor que seja seu foco em termos de viabilidade, propriedade e precisão" (Worthen et al., 2004, p. 596).

Avaliações que proporcionam reflexão crítica sobre a prática pedagógica e seu compromisso com o crescimento dos alunos, professores e escolas brasileiras são cada vez mais necessárias e urgentes. Para Hoffmann (1998), a consciência "da natureza política da avaliação e a compreensão da dimensão que essa política tem assumido na prática escolar deveriam ser ponto de partida para reconduzir a avaliação às suas reais funções". Uma avaliação do ensino, conduzida de forma útil, viável, precisa e ética, poderá subsidiar novos estudos sobre a adequação da formação, apontando caminhos para as necessárias atualizações e reformulações curriculares.

\section{Análise dos Resultados}

Para assegurar o alcance dos objetivos traçados para o estudo, buscamos responder a seguinte questão: a partir dos dados coletados, é possível comparar os resultados da avaliação global do CIASC, diagnosticando avanços e melhorias na qualidade do ensino e da gestão da instituição, ao longo de três anos de aplicação da política de avaliação vigente na MB?

Importante ressaltar que a análise comparativa dos resultados da avaliação global do ensino no CIASC, obtidos com a aplicação da metodologia de avaliação do SEN, de 2007 a 2009, se fez com base em documentos oficiais da OM, encaminhados anualmente à DEnsM, por ofício.

Observamos que o resultado global alcançado pelo estabelecimento de ensino na primeira avaliação, referente ao ano base 2006, constante do Relatório de Avaliação Interna (RAInt) de 2007, foi de 762 pontos, equivalente ao conceito Bom. No quadro resumo da avaliação, verificamos alguns comentários da OM sobre as dimensões avaliadas, indicando uma preocupação em atender os parâmetros exigidos nos instrumentos avaliativos previstos. 
Com a avaliação realizada, ficou evidente que a Dimensão Instalação era a maior dificuldade da $\mathrm{OM}$, pois obteve conceito Insuficiente. No campo comentário, a OM aponta que há necessidade de investimento em recursos instrucionais, material didático e laboratórios. A partir dos resultados expressos neste relatório, foi possivel diagnosticar os potenciais de crescimento da $0 M$, para se trabalhar em prol das melhorias necessárias.

No RAlnt de 2008, referente ao ano base 2007, a OM obteve uma evolução de 47 pontos na avaliação global, saltando de 762 pontos para 809 pontos, porém, mantendo-se na faixa de pontuação que equivale ao conceito Bom. Na parte destinada ao Parecer Geral e Recomendações, a OM registrou que um planejamento a curto e médio prazos seria elaborado, visando o aperfeiçoamento dos aspectos com possiveis pontos de melhoria, particularmente nas dimensões Instalações e Corpo Docente, que receberam conceitos Insuficiente e Regular, respectivamente. Além disso, foi possivel identificar, no próprio relatório, que as discussões em torno da avaliação se intensificaram na $0 \mathrm{M}$, com a realização de três reuniões de Conselho de Ensino, ocorridas nos meses de março, junho e dezembro de 2007.

Consideramos o Conselho de Ensino um fórum propício para a discussão coletiva dos resultados alcançados, porque é necessário o envolvimento de todos, com múltiplos enfoques, definindo o que é preciso realizar para superar as "fraquezas", sem melindres e constrangimentos, de forma ética.

A pouca participação e envolvimento dos membros de uma instituição avaliada podem ser ocasionados por vários motivos, dentre eles, a falta de informação e divulgação do propósito da avaliação que se deseja realizar; a interpretação equivocada de que a avaliação tem caráter regulador e punitivo; e o descrédito pelos benefícios que o processo avaliativo poderá trazer para a instituição e seus membros. Portanto, para o sucesso de um empreendimento avaliativo é preciso que ele seja bem aceito e que promova "a participação intensa de seus membros, tanto nos procedimentos e na implementação, como na utilização dos resultados" (RISTOFF, 1996, p. 52-53). A credibilidade dos elementos envolvidos na avaliação que se pratica é fundamental.

Além das portarias de designação de militares para comporem a Comissão de Avaliação de Cursos (CAC), verificamos que o CIASC designou, também por portaria, militares para compor a Comissão de Avaliadores do Corpo Docente e para compor a Comissão de Informantes Qualificados da Avaliação Institucional. Observamos ainda, que o número de participantes da CAC aumentou gradativamente. Em 2007, a OM designou quinze militares e um funcionário civil para compor a CAC, totalizando dezesseis membros. Em 2008, este número aumentou para dezenove e, em 2009, para vinte e um. 
Vimos como positiva a iniciativa de designar os informantes qualificados por portaria porque, além de estabelecer responsabilidades a cada respondente da avaliação, pôde garantir o que Ristoff $(1996$, p. 56) chama de legitimidade técnica do processo avaliativo, pois a "existência de uma base de dados confiáveis é também condição necessária para qualquer esforço de avaliação que se proponha".

No RAInt 2009 do CIASC, referente ao ano base 2008, observamos que a OM obteve 934 pontos na avaliação global, apresentando progressos em relação ao RAlnt 2008. Analisando comparativamente os RAlnt dos três anos, observamos que no RAInt 2009, houve melhorias significativas. Dentre elas, podemos destacar duas, que foram o aumento do número de docentes que apresentaram ou publicaram trabalhos e/ou artigos e o aumento de docentes que participaram de eventos de capacitação, evidenciando a preocupação com o desempenho acadêmico e com os estímulos profissionais do corpo docente da OM.

Outro aspecto que merece destaque registrado no RAlnt 2009 foi a realização de seis reuniões do Conselho de Ensino no ano de 2008, sendo cinco delas relacionadas aos assuntos abarcados na avaliação institucional. A primeira, em fevereiro, para a apresentação do RAInt; a segunda, em março, para homologação dos resultados da avaliação interna e para a elaboração do parecer geral no RAlnt; a terceira, em maio, para analisar os currículos dos cursos; a quarta, em junho, para organização dos procedimentos para melhoria do ensino; e a quinta, realizada em setembro, para tratar da avaliação do ensino e do Programa de Capacitação e Qualificação de Docente (PCQD). Essa prática se coaduna com o pensamento de Penna Firme e Letichevsky (2002, p. 290), quando afirmam que quanto maior e mais significativo o envolvimento dos interessados pela avaliação, maior será o uso dos resultados.

No mês de junho de 2009, o CIASC recebeu a Comissão de Avaliação do SEN (CAS), instituída pelo CPesFN, para realização da Avaliação Externa. Em resposta ao Relatório de Avaliação Externa (RAExt) 2009, o CIASC estabeleceu algumas medidas a curto e médio prazos, definidas por dimensão avaliada. Estas medidas são denominadas de ações decorrentes da avaliação no âmbito do SEN e traduzem os aspectos que serão aprimorados pelos gestores da $\mathrm{OM}$ de ensino a partir do diagnóstico realizado, evitando que o relatório da avaliação permaneça na esfera contemplativa.

Ao defenderem a importância da meta-avaliação, Penna Firme e Letichevsky (2002, p. 297) explicam que o "ciclo avaliativo é uma continuidade permanente, em busca de seu aperfeiçoamento e de consequentes repercussões. É assim que o processo se institucionaliza". 0 estabelecimento de metas estimula a busca por melhores resultados e dá um sentido de continuidade do processo avaliativo.

Worthen et al. (2004, p. 38) alertam sobre as limitações potenciais da avaliação, que por si só não cria soluções. A avaliação, na verdade, não resolve os problemas 
detectados, mas aponta caminhos para correções de rumo. Neste sentido, procuramos identificar a seguir, as ações decorrentes e metas estabelecidas pelo CIASC, a partir das considerações da comissão externa, em busca da excelência do ensino.

Na Dimensão Corpo Docente, as principais medidas estabelecidas a curto prazo foram: a) realizar um trabalho de Orientação Pedagógica com os instrutores que receberam conceitos Bom, Regular e Insuficiente nas avaliações de aula; b) manter permanentemente a capacitação pedagógica dos docentes; c) providenciar 0 comparecimento de um número maior de docentes em eventos de capacitação; e d) ampliar o número de instrutores ministrando uma só disciplina por vez, por meio de alterações nas grades curriculares.

Dentre as medidas estabelecidas a médio prazo destacamos as seguintes: a) realizar encontros mensais que possibilitem trabalhar questões motivacionais e de relações humanas e liderança, assim como atender as necessidades dos instrutores, sempre que possivel; e b) realizar reuniões para estudo de cursos que possam contribuir para a qualificação técnica dos docentes.

Na Dimensão Corpo Discente, foi definida como medida a curto prazo, um maior intercâmbio entre os departamentos da $\mathrm{OM}$, permitindo um acompanhamento mais eficaz do desempenho do instrutor durante a transmissão do conteúdo da disciplina, assim como um monitoramento mais efetivo das notas dos discentes por meio dos quadros de apuração. A médio prazo, foi estabelecida como medida, manter o horário destinado às atividades de monitoria, para todas as turmas, selecionando os alunos com maior aproveitamento na disciplina e criando mecanismos de recompensa para estes.

Na Dimensão Organização Didático-Pedagógica, foram registradas as ações já implementadas na $\mathrm{OM}$ como, cumprir integralmente as cargas horárias das disciplinas e empregar na função de coordenador, militares que tenham experiência no tipo de ensino ou no exercício profissional da área.

Na Dimensão Instalações, foi estabelecida como meta a curto prazo, empregar os equipamentos existentes em prol dos cursos. A médio prazo, foram estabelecidas como metas: a) prontificar os sanitários e alojamentos dos docentes e discentes; e b) realizar melhorias nas instalações administrativas. A OM registrou também que estavam sendo realizadas obras nos alojamentos dos alunos e nos da guarnição, onde seria construída uma área de lazer para eles.

Pelo acima exposto, verificamos que a OM considerou, de forma pró-ativa, as recomendações do RAExt, estabelecendo as ações decorrentes da avaliação de 2009 de modo a perseguir melhorias. Os aspectos que ainda necessitam ser trabalhados podem ser encarados como os potenciais de crescimento. 
0 valor da avaliação contínua, defendida por Ristoff $(1996,2000)$, está na possibilidade de comparar os resultados históricos da $\mathrm{OM}$, verificando o que evoluiu e o que permaneceu. Nesse sentido, a avaliação será uma caminhada diária e necessária, em sintonia com o que preceitua o Manual DEnsM-2001.

É importante que a avaliação tenha um efetivo caráter formativo, à medida que orienta quanto à necessidade de adoção de novas estratégias, capazes de gerar elementos que facilitem a superação dos problemas detectados, propiciando o diálogo entre todos os usuários do SEN (BRASIL, 2011, p.1-2).

Ao se realizar uma meta-avaliação, no que se refere à categoria utilidade, é necessário investigar se as informações práticas chegam ao usuário, por meio de relatórios que são divulgados a tempo de provocar mudanças, favorecendo a capacitação dos avaliadores em avaliar. Observamos que isso é possível por meio da divulgação do RAExt, elaborado após a Avaliação Externa na OM de ensino. Internamente, a divulgação do RAlnt também pode ser realizada na $\mathrm{OM}$ para que todos os elementos participantes do processo avaliativo percebam as contribuições da metodologia de avaliação, num processo de retroalimentação.

Quanto à necessidade de capacitação dos avaliadores, a MB define que "compete à DEnsM a promoção de cursos ou treinamentos que possibilitem a capacitação dos avaliadores" (BRASIL, 2011, p. 1-6). Portanto, consideramos algo a ser proporcionado com o tempo, à medida que o manual DEnsM-2001 for sendo utilizado nas OM de ensino do SEN.

A Diretoria de Ensino da Marinha (DEnsM) pretende, ao capacitar tecnicamente avaliadores, modificar a visão negativa que alguns têm da avaliação, relacionando-a a sentimentos de punição e fracasso. Diminuir as resistências em relação à avaliação, para que o avaliado não se sinta inibido e/ou constrangido, certamente torna-se passo importante para o sucesso de uma avaliação [...]. (AGUIAR; CANEN, 2007, p. 58)

É importante que o avaliador tenha conhecimento das diferentes abordagens avaliativas, tenha clareza do por que, para quê, e de como avaliar. Se não há certeza do que se quer, a avaliação pouco contribuirá.

A capacitação de profissionais para o exercício da avaliação e a expansão do conhecimento e da prática na área, para atingir, democraticamente, todos os envolvidos e interessados no desenvolvimento e no aperfeiçoamento da qualidade do foco da avaliação, é o desafio por excelência nessa entrada no século XXI. Acima de tudo, porém, é a qualidade da avaliação que vai garantir em última análise o sucesso da avaliação da qualidade. (PENNA FIRME; LETICHEVSKY, 2002, p. 290). 
Uma nova cultura de avaliação se faz necessária e só será possivel, segundo Vasconcellos (1998), com um amplo processo de busca de ressignificação teórica e prática. Neste contexto, o autor afirma que construir um sentido novo, uma nova intencionalidade para a avaliação é decisivo.

\section{Conclusões}

0 argumento de que a avaliação é uma necessidade para qualquer esforço de melhoria vem sendo amplamente defendido por estudiosos da educação, que pesquisam sobre a avaliação institucional e a avaliação de programas, assim como definido no Manual de Avaliação do SEN. Os fundamentos teóricos apresentados nesta pesquisa reforçam a importância da continuidade do processo avaliativo e da meta-avaliação, avaliando a própria avaliação que se realiza, verificando seu mérito e relevância.

0 estudo da meta-avaliação utiliza quatro grandes categorias ou padrões, que são utilidade, viabilidade, ética e precisão. Para este trabalho, optamos pela categoria utilidade para as análises realizadas. Com o propósito de verificar se a nova metodologia de avaliação, implementada na Marinha do Brasil desde 2006, tem sido útil, foram analisados os resultados da Avaliação Interna e Externa do CIASC, apresentados nos RAInt e RAExt da OM, de 2007 a 2009, para diagnosticar avanços e melhorias na qualidade do ensino e da gestão da instituição.

A análise comparativa das pontuações da avaliação global do CIASC permitiu verificar que a $\mathrm{OM}$ apresentou uma evolução dos seus resultados, tendo uma melhoria contínua em sua pontuação. 0 Centro de Instrução pesquisado evoluiu de 762 pontos, alcançados em sua primeira avaliação, no ano de 2007, para 934 pontos, em 2009.

Além da identificação do progresso em termos de pontuações alcançadas pelo CIASC, o estudo permitiu verificar um comprometimento da OM em termos de definição de metas, a curto e médio prazos, a partir da análise qualitativa dos aspectos apontados pela CAS na Avaliação Externa conduzida pelo CPesFN em 2009. As principais metas estabelecidas foram para as Dimensões Corpo Docente e Instalações. Além disso, evidenciamos o aumento de reuniões na OM para tratar dos assuntos afetos à avaliação do ensino, como a capacitação dos docentes, a reformulação dos currículos dos cursos, e o estabelecimento de estratégias e procedimentos de melhoria dos resultados alcançados.

Esta pesquisa possibilitou, então, verificar que a aplicação da metodologia de avaliação do SEN, DEnsM-2001, atual política avaliativa do ensino na MB, tem contribuído para a melhoria da qualidade do ensino no CIASC, sendo uma ferramenta útil e eficaz na busca contínua por padrões de excelência no ensino, cumprindo o propósito para o qual foi criada. Observamos também que a participação dos elementos envolvidos no empreendimento avaliativo, a atitude e mentalidade de 
busca pela constante melhoria em prol da formação militar e da qualidade dos processos que envolvem o ensino conduzido na $0 \mathrm{M}$, são os verdadeiros ganhos proporcionados pela prática avaliativa em tela.

Numa perspectiva mais ampla, esta pesquisa abre caminhos para novas discussões sobre o tema da avaliação institucional e traz experiências positivas e inovadoras para o avanço de futuras práticas avaliativas em instituições de ensino, civis ou militares, e para a construção de outras metodologias de avaliação, adaptadas às distintas realidades institucionais.

\section{Referências}

AGUIAR, N. M. C. B.; CANEN, A. Impactos de políticas de avaliação institucional: um estudo de caso no sistema de ensino naval brasileiro. Ensaio: avaliação e políticas públicas em educação, Rio de Janeiro, v. 15, n. 54, p. 53-66, jan./mar. 2007.

BRASIL. Lei $n^{\circ}$ 11.279, de 09 de fevereiro de 2006. Dispõe sobre o ensino na Marinha. Diário Oficial [da] República Federativa, Brasília, DF, 10 de fevereiro de 2006.

Diretoria de Ensino da Marinha. DEnsM-2001: Manual de Avaliação do Sistema de Ensino Naval (2a rev.), Rio de Janeiro, 2011.

CANEN, A. Avaliando a avaliação a partir de uma perspectiva multicultural. Educação Brasileira: revista do CRUB, Brasilia, DF, v. 27, n. 54, p. 95-114, 2005.

DIAS SOBRINHO, J. Avaliação institucional, instrumento da qualidade educativa: a experiência da Unicamp. In: BALZAN, N. C.; DIAS SOBRINHO, J. (Org.). Avaliação institucional: teoria e experiências. 2. ed. São Paulo: Cortez, 2000. p. 53-86.

HOFFMANN, J. M. L. Contos e contrapontos: do pensar e agir em avaliação. Porto Alegre: Mediação, 1998.

LUCKESI. C. C. Avaliação da aprendizagem escolar: estudos e proposições. São Paulo: Cortez, 1995.

PENNA FIRME, T.; LETICHEVSKY, A. C. 0 Desenvolvimento da capacidade de avaliação no Século XXI: enfrentando o desafio através da Meta-Avaliação. Ensaio: avaliação de políticas públicas em avaliação, Rio de Janeiro, n. 35, v. 10, p. 289-300, 2002.

RISTOFF, D. I. Princípios do Programa de Avaliação Institucional. Avaliação: Revista de Avaliação Institucional das Instituições de Ensino Superior, Campinas, n. 1, 1996, p. 47-68 68. 
Avaliação institucional: pensando principios. In: BALZAN, N.C.; DIAS SOBRINHO, J. (Org.). Avaliação institucional: teoria e experiências. 2. ed. São Paulo: Cortez, 2000. p. 37-51.

VASCONCELLOS, C. S. Avaliação: Superação da Lógica Classificatória e Excludente - do "é proibido reprovar" ao é preciso garantir a aprendizagem. São Paulo:

Libertad, 1998.

YARBROUGH, D. B. et al. The program evaluation standards: a guide for evaluators and evaluation users. 3rd ed. Thousand Oaks, Califórnia: Sage Publications, 2011.

WORTHEN, B. R.; SANDERS, J. R.; FITZPATRICK, J. L. Avaliação de Programas: concepções e práticas. Trad. Dinah de Abreu Azevedo. São Paulo: Editora Gente; Edusp, 2004.

Recebido em: 18/05/2013

Aceito para publicação em: 05/02/2013

\section{Policy evaluation of naval education Abstract}

This article is a case study on evaluation of Navy Education and analyzses contributions of an institutional evaluation experience in the Brazilian Navy. It analyzes the contributions of Evaluation Methodology of the Naval Education System, a comparative perspective of the results achieved within three years of its implementation in CIASC. The research analyzes the institutional evaluation and meta-evaluation concepts and "evaluation culture" and its theoretical studies are Penna Firme and Letichevsky (2002), Dias Sobrinho (2000) and Ristoff $(1996,2000)$. Data show that the quality of teaching has improved with progressively higher scores and it was found that meetings to discuss issues relating to evaluation have become more frequent in the organization of teaching, in which targets were set for continuous improvement in pursuit of excellence. This research opens the way for the construction of new models of institutional assessment, tailored to different academic realities.

Keywords: Meta-evaluation. Navy Education. Teaching.

\section{Política de evaluación de la educación naval Resumen}

Este artículo es un estudio de caso sobre la evaluación de la enseñanza naval y analiza las contribuciones de una experiencia de evaluación institucional en la Marina de Brasil. En él se observan las contribuciones de la Metodología de 
Evaluación del Sistema de Enseñanza Naval, bajo una perspectiva comparativa de los resultados obtenidos en los tres años de su aplicación en el CIASC - Centro de Instrucción Almirante Sylvio de Camargo.

La investigación analiza la evaluación institucional y los conceptos de la metaevaluación y de la "cultura de evaluación" y su fundamentación teórica son los estudios de Penna Firme y Letichevsky (2002), Dias Sobrinho (2000) y Ristoff (1996, 2000). A partir de los análisis, llegamos a la conclusión de que la calidad de la enseñanza naval ha mejorado con el alcance progresivo de puntajes cada vez más elevados y verificamos que las reuniones para discutir cuestiones relativas a la evaluación son, actualmente, más frecuentes en la organización de la enseñanza, y con ello se fijaron los objetivos para la mejora continua en búsqueda de excelencia. Esta investigación abre camino para la construcción de nuevos modelos de evaluación institucional, adaptados a las diferentes realidades académicas.

Palabras clave: Metaevaluación. Enseñanza naval. Enseñanza. 